\title{
Candida as Pathogens of Onychomycosis among Elderly Diabetic Patients
}

\author{
Warda MB Bridan ${ }^{1 *}$, Saleh H Baiu², Hanan MK Kalfa ${ }^{3}$ \\ ${ }^{1}$ Department of Microbiology, Libya \\ ${ }^{2}$ Department of Botany, Libya \\ ${ }^{3}$ Department of Dermatology, Libya \\ *Corresponding author: Warda M B Bridan, Department of Microbiology, Libya \\ Submission: October 11, 2018; Published: December 11, 2018
}

\begin{abstract}
Onychomycosis is a common fungal infection affecting nails. The primary cause for onychomycosis is Non-dermatophytes, while Candida species have emerged as second-line pathogens. Onychomycosis due to Candida [candidal onychomycosis] is increasingly found in i patients with diabetes mellitus (T2DM). Diabetes can result in complications that affect all systems of the body. Of particular relevance to onychomycosis rates is the effect of (T2DM). on the lower extremities. (T2DM). can lead to lower extremity arterial disease; in turn this can predispose patients to onychomycosis of the toenails.
\end{abstract}

Materials and methods: Incidence were determined in 116 type 2 diabetes mellitus (T2DM) patients over from September2013 to January 2014 included direct microscopy and repeated cultures. A higher incidence of onychomycosis. who were registered at the Sedee Hussein Polyclinic of Benghazi city.

Results: The prevalence of onychomycosis among diabetics in our study was high, culture was positive in 84 of 116 patients [41\%] patients with onychomycosis, laboratory culture identified yeasts as the pathogen in $22 \%$ of positive cases.

Conclusion: Onychomycosis is an important cause of morbidity in diabetic patients, increasing their risks for limb amputation and local and systemic secondary bacterial infections. Because onychomycosis is more common in diabetic patients and can complicate the disease, clinicians must be vigilant in its diagnosis and complete in its treatment.

Keywords: Onychomycosis; Non- dermatophytes; Candida; Toenails; Species; Type 2 diabetes mellitus

\section{Introduction}

Patients with diabetes represent a unique group of individuals who appear more prone to develop infections than others. Several mechanisms have been proposed to explain the association between diabetes and infections. However, few conclusive studies exist, and a considerable debate is going on regarding the evidence for this predisposition. Diabetes mellitus is a chronic disorder that affects a large segment of the human population and is a major public health problem Diabetes and foot problems are almost synchronous [1-3].

Diabetic foot infections frequently result in morbidity, hospitalization and amputations. The bacteriology of diabetic foot ulcers has been studied by numerous investigators [4-9]. The present study was undertaken in order to evaluate the incidence of pathogenic fungi.

\section{Materials and Methods \\ Collection of samples}

Two hundred and two T2DM patients were enrolled in the study. Excluding criteria were patients on systemic antifungal agents for 3 month or on topical agents during one month prior to the study Patients samples were taken from patients directly by clipping the nail after cleaning the affected areas with $70 \%$ alcohol.

\section{Derect microscopic examination}

The collected specimen was placed in a test tube [Assistant, Germany] and fews drops of $\mathrm{KOH}$ [20\% solution] were added using eye dropper to the glass tube and kept for 24 hours to dissolve

the keratin. The collected specimen is then placed on a glass slide [Hamburrg - Germany], and covered by a cover glass [HamburrgGermany]. Repeated KOH examination was performed before the specimen was considered as negative for direct microscopic mount.

\section{Cultivation of the specimens}

The specimen of each patient was placed in separate sterile Petri dish. Each specimen was inoculated on sabouraud`s dextrose Agar [SDA], and Fungobiotic agar. The inoculated plates were kept in the incubator [MMM- Grafelfing, Germany] which was adjusted at $28{ }^{\circ} \mathrm{C}$ and the cultures were examined every two days. The culture 
was considered negative if there was no growth after four weeks of incubation. The positive specimens [fungi cultures] were mounted with the lactophenol cotton blue to reveal various structures which could be of great help in identification, especially the conidia which include the large separated macroconidia and the small celled microconidia. The macroconidia of each genus and species vary in shape and character of their walls which are generally characteristic for the species or genus.

The identification of Candida was based on the presence of budding cells and pseudohyphae. BD PHOENIX [Becto, Dickinson / USA] is an automated microbiology system intended for the in vitro rapid identification [ID] of yeast and yeast like organisms.

\section{Statistical Analysis}

Frequency tables and chart constructed for our data were analyzed statistically using the chi-square test. We assumed results statistically significant when $\mathrm{P}$ value is $<0.005$. The statistical analysis of the results were carried out according to the computer package [SPSS 18.0 version].

\section{Results}

The study included each 116 diabetic patients from type 2 diabetes mellitus (T2DM) from all patients who were registered at the Sedee Hussein Polyclinic of Benghazi city. diabetes patients, 35 were males and 49 females. The age wasover 60 years to 93 diabetes patients. Direct microscopic analysis using $\mathrm{KOH}$ was positive in $72[62 \%]$ specimens example, and negative in 44 [38\%]. Among the fungal cultures identified, yeast [31\%, $\mathrm{n}=26$ ], Non- dermatophytes molds isolated [ $57 \%, \mathrm{n}=48]$, dermatophyte [5\%, $\mathrm{n}=4]$ and mixed fungi $[7 \%, \mathrm{n}=6]$.

Candida was more frequent in age from 60 to 65 the distribution was similar in both female and male genders [Table1] depicts the identification scheme for yeast cultures adopted in this study. Candida parapsilosis complex, was the dominant organism causing onychomycosis 8 [31\%], followed by Trichosporon asahii 6 [23\%] Candida albicans 3 [11\%], Trichosporon inkin 2[8\%], and Candida tropicalis 2 [8\%], Candida was the major isolated species.

Table 1: Candida species isolated from diabetic T2DM diabetic.

\begin{tabular}{|c|c|c|}
\hline Candida species & Total & $\mathbf{\%}$ \\
\hline Candida parapsilosis & 8 & 31 \\
\hline Trichosporon asahii & 6 & 23 \\
\hline Candida albicans & 5 & 19 \\
\hline Trichosporon inkin & 3 & 11 \\
\hline Candida tropicalis & 2 & 8 \\
\hline Candida rugosa & 2 & 8 \\
\hline
\end{tabular}

\section{Discussion}

Dermatophytes are the predominant pathogens in temperate Western countries; meanwhile, Candida and non-dermatophyte moulds are prevailing in Mediterranean and tropical countries.
Clinicians should appreciate that the pathogens of onychomycosis may vary in different patient groups and geographical locations and choose optimal antifungal agents accordingly. The T2DM patient onychomycosis in our study was predominant in toenails and the distribution was similar in both female and male genders. Factors that can contribute to the increased prevalence of onychomycosis in the geriatric population include a reduced rate of nail growth and a greater frequency of trauma with the consequence of a more propitious opportunity for causing disease in the nail bed and plate [10-12].

In our study, the same health conditions were mentioned by a high percentage of patients. Onychomycosis contributes to the severity of diabetic foot problems $[13,14]$. Sharp, brittle nails can gouge the skin, creating a portal for entry of bacterial organisms. Onychomycosis is often associated with tinea pedis, which can create fissures in the skin, again opening the way for bacterial infections. Thickened mycotic nails can cause pressure necrosis of the nail bed These injuries may go unnoticed in patients with impaired sensation due to peripheral neuropathy. Because of the proximity of the nail bed to underlying bone, osteomyelitis can develop because of neglected nail bed erosion [14].

In a retrospective study of outpatient medical care claims, onychomycosis was associated with a threefold increase in gangrene and foot ulcers among patients with diabetes [15]. The presence of onychomycosis can contribute to the difficulties of maintaining nail hygiene in elderly diabetic patients. Onychomycosis can also contribute to self-consciousness, lowering of self-esteem, and restriction of social activities in younger diabetic individuals [11].

In this study, age greater than 60 years was significantly associated with presence of onychomycosis. Other studies have also reported a higher prevalence of onychomycosis among the elderly [10,16-18]. In this study was Candida species have emerged as second-line pathogens. Candida was more frequent in age from 60 to 65 the distribution was similar in both female and male genders. This is probably because other factors, such as environment, level of humidity and the repeated contact with water influences the growth of particular fungi $[19,20]$.

However, in one Indian study, yeasts were found to be the most common pathogens causing onychomycosis in diabetic patients, followed by dermatophytes and non-dermatophyte moulds [16]. Another study from Saudi Arabia reports on Candida species as the most frequently isolated pathogen from infected nails [21]. In Kuwait, dermatophytes were the most common isolates causing onychomycosis in diabetic patients, followed by yeasts and nondermatophyte moulds [16].

This type of fungal infection is more common in fingernails than toenails. It may involve all of the nails at the same time and can cause the nail to separate from the nail bed. It invades weakened areas of the nail.

\section{Conclusion}

Diabetic patients are at increased risk of developing onychomycosis. As the population of diabetic subjects continues to 
grow, it is likely that clinical and economic impact of onychomycosis in these individuals the will increase. The increasing incidence, varied epidemiology and the physical and psychological ramifications of the disease emphasize the need for effective broad-spectrum treatment in susceptible patient Therefore recognition and early intervention is advisable because of the potential progressive nature of fungal, populations infections and the potentially serious sequels associated with persistence of untreated infected nails. The consequences of neglecting onychomycosis may carry more risk for diabetics compared with nondiabetic patients. Although it is the responsibility of the clinician to accurately diagnose and pertinently treat onychomycosis, education of diabetic patients about the importance of foot and nail care should form an essential component of diabetes management. This is especially important in-patient groups at a higher risk for the development of onychomycosis, such as elderly diabetics..

\section{References}

1. Chander J (1995) A text book of medical mycology (3 $3^{\text {rd }}$ edn). Inter print, New Delhi, India.

2. Frykberg RG (1998) J Foot Ankle Surg 37(5): 440-446.

3. Shea KW (1999) Postgrad Med 106(1): 85-94.

4. Bamberger DM, Daus GP, Gerding DN (1985) Amer J Medicine 83: 653660.

5. Gerding DN (1995) Clin Infect Dis 20: S283-S288.

6. Lipsky BA, Pecoraro RE, Larson SA (1990) Arch Intern Med 150: 790797.

7. Peterson LR, Lissack LM, Canter K (1989).

8. Sharp CS, Bessman AN, Wagner W (1979) Surg Gynecol Obstet 149: 217 219.

9. Wheat LJ, Allen SD, Henry M (1986) Arch Intern Med 146: 1935-1940.
10. Gupta AK, Konnikov N, MacDonald P, Rich P, Rodger NW, et al. ( 1998 ) Prevalence and epidemiology of toenail onychomycosis in diabetic subjects: A multicentre survey. Br J Dermatol 139(4): 665-671.

11. Rich P (1996) Special patient populations onychomycosis in the diabetic patient. J Am Acad Dermatol 35(3): S10-S12.

12. Arrese JE, Piérard GE (2003) Treatment failures and relapses in onychomycosis: A stubborn clinical problem. Dermatology 207: 255260.

13. Greene RA, Scher RK (1987) Nail changes associated with diabetes mellitus. J Am Acad Dermatol 16: 1015-1021.

14. Rich P, Hare A (1999) Onychomycosis in a special patient population: focus on the diabetic. Int J Dermatol 38(Suppl 2): 17-19.

15. Boyko WL, Doyle JJ, Ryu S, Gause D (1999) Onychomycosis and its impact on secondary infection development in the diabetic population (Poster). Presented at the $4^{\text {th }}$ annual meeting of the International Society for Pharmacoeconomics and Outcomes Research (ISPOR), Arlington. VA, USA.

16. Dogra S, Kumar B, Bhansali A, Chakrabarty A (2002) Epidemiology of onychomycosis in patients with diabetes mellitus in India. Int J Dermatol 41(10): 647-651.

17. Chang SJ, Hsu SC, Tien KJ, Hsiao JY, Lin SR, et al. (2008) Metabolic syndrome associated with toenail onychomycosis in Taiwanese with diabetes mellitus. Int J Dermatol 47(5): 467-472.

18. Saunte DM, Holgersen JB, Haedersdal M, Strauss G, Bitsch M, et al. (2006) Prevalence of toenail onychomycosis in diabetic patients. Acta Derm Venereol 86(5): 425-428.

19. Kafaie P, Noorbala MT (2010) Evaluation of onychomycosis among diabetic patients of yazd diabetic center. J Pakistan Assoc Dermatol 20: 217-221.

20. Berker D (2009) Clinical practice: Fungal nail disease. N Engl J Med 360(22): 2108-2116.

21. Sogair SM, Moawad MK, Al-Humadan YM (1991) Fungal infection as a cause of skin disease in the eastern province of Saudi Arabia: prevailing fungi and pattern of infection. Mycoses 34(8): 333-337.
Creative Commons Attribution 4.0

International License

For possible submissions Click Here

\section{Submit Article}

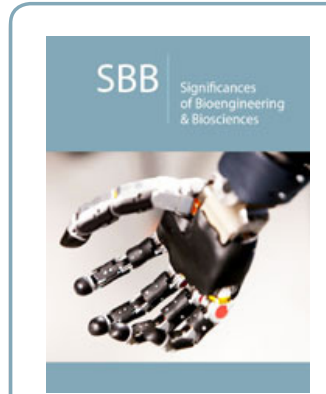

Significances of Bioengineering \& Biosciences

\section{Benefits of Publishing with us}

- High-level peer review and editorial services

- Freely accessible online immediately upon publication

- Authors retain the copyright to their work

- Licensing it under a Creative Commons license

- Visibility through different online platforms 